\title{
Understanding Potential Intraoperative Impediments for Learning Laparoscopic Nephrectomy
}

\author{
Alon Z. Weizer, M.D., ${ }^{1,2}$ Zaojun Ye, ${ }^{3}$ J. Stuart Wolf, Jr., M.D., ${ }^{1}$ and Brent K. Hollenbeck, M.D. ${ }^{1,2,3}$
}

\begin{abstract}
Purpose: We evaluated factors that impact the ability to learn hand-assisted laparoscopic donor nephrectomy (HALDN) to identify impediments to diffusion of this procedure.

Methods: From February 2002 to June 2004, we collected data from our institutional database on 70 patients who underwent HALDN. Time for individual steps of the procedure (colon mobilization, kidney/ureter mobilization, renal vein tributary dissection, renal hilum dissection, removal of the kidney, and overall time) were recorded. The impact of patient factors on surgical times was assessed using a general linear model. The impact of individual operative steps on overall operative time was assessed using Pearson correlation. The influence of case experience and training level were evaluated graphically and in a multivariable model.

Results: A total of 13 residents, 2 fellows, and 1 attending surgeon participated in procedures for 70 patients. Body mass index $(P=0.03)$ and male sex $(P=0.04)$ prolonged operative times. Colon mobilization and hilar dissection were most correlated with overall operative time. While experience improved operative times for several steps, level of training appeared more likely to influence the time for individual operative steps.

Conclusions: Impediments to learning HALDN include patient factors, level of training, and particular surgical steps. Repeated exposure at increasing levels of training may improve diffusion of laparoscopic nephrectomy among urologists.
\end{abstract}

\section{Introduction}

A BURGEONING LITERATURE demonstrates that laparoscopic approaches are the preferred technique for the surgical management of many benign and malignant diseases affecting the kidney. ${ }^{1-3}$ For example, compared with open radical nephrectomy for organ-confined renal masses, laparoscopic nephrectomy has resulted in equivalent oncologic outcomes and complication rates with shorter convalescence and improved cosmesis. ${ }^{4}$

Despite these benefits, a recent study has demonstrated that laparoscopic nephrectomy has been slow to diffuse compared with laparoscopic procedures performed by other specialties introduced during the same period. ${ }^{5}$ In addition, while other procedures, such as laparoscopic cholecystectomy, are the predominant approach to the removal of the gallbladder, laparoscopic nephrectomy is the approach in only $25 \%$ of the patients undergoing kidney removal. Laparoscopic donor nephrectomy, however, performed by urologists as well as transplant surgeons has seen a more rapid diffusion compared with other indications for kidney removal. ${ }^{6}$

The dichotomy between the benefits of this approach for the patient and the slow diffusion among the group of surgeons most likely to perform this procedure has led us to evaluate potential factors that influence the learning of laparoscopic nephrectomy. Specifically, we evaluated the impact of patient and surgeon factors on operative times for individual steps of hand-assisted laparoscopic donor nephrectomy (HALDN) to identify impediments to diffusion of this procedure.

\section{Methods}

After Institutional Review Board approval, 70 consecutive patients undergoing HALDN from February 2002 to June 2005 who were directly supervised by a single surgeon (JSW) were enrolled. Routine medical, surgical, and psychosocial preoperative evaluation was performed to assess subjects' suitability as a renal donor.

Divisions of ${ }^{1}$ Minimally Invasive Surgery, ${ }^{2}$ Urologic Oncology, and ${ }^{3}$ Health Services Research, Department of Urology, University of Michigan, Ann Arbor, Michigan. 
Our technique for HALDN has been previously described. ${ }^{3}$ Time for individual surgical steps (colon mobilization, kidney/ureter mobilization, renal vein tributary dissection, renal hilum dissection, removal of the kidney, overall time), and surgeon responsible for each step (individual experience and level of training) were collected prospectively at the time of the surgical procedure. Specifically, junior residents were those within their first year of urology training, senior residents were those in their third or fourth year of urology training, fellows were those having completed a urology residency participating in a Endourological Society approved fellowship program, and an attending was the person who was supervising the surgery. Patient age, sex, American Society of Anesthesiologists (ASA) class, body mass index (BMI), previous intraabdominal surgery, nephrectomy side, and abnormal vasculature as determined by preoperative imaging or intraoperative findings were abstracted from the patients' medical records.

The impact of patient factors (above) on surgical times (each step and overall) was assessed using a general linear model. A Pearson correlation coefficient was used to evaluate the correlation of operative time of individual steps to overall operative time. Surgical times (median, 25th and 75th percentile, maximum and minimum times) were plotted by case number for individual steps to evaluate the influence of case experience on surgical times.

A general linear model was fit to measure the association of training level on operative times, adjusting for patient factors. For this analysis, only junior and senior resident operative times for overall operative times, colon mobilization, and kidney mobilization were compared, because the other steps were performed predominantly by fellows or the attending with inadequate numbers of surgeons to compare between groups. All statistical analysis was performed using SAS v 9.2 (SAS Institute, Cary, NC) and carried out at the $5 \%$ significance level.

\section{Results}

A total of 70 consecutive patients undergoing HALDN were enrolled in the study. Median age was 42 years (range 19-67), and median BMI was $27.4 \mathrm{~kg} / \mathrm{m}^{2}$ (range 19.8-37.6). All patients were either ASA class 1 or 2 , and $60 \%$ of the patients were women. A total of 13 residents (6 junior residents, 7 senior residents), 2 fellows, and 1 attending surgeon participated in these procedures.

To understand the influence of patient factors on overall operative time, we identified several patient variables that might influence the length of the operative procedure. Table 1 evaluates the impact of patient factors on the time of individual steps as well as overall operative time.

Of all evaluated factors, only sex and BMI influenced operative times in our cohort of patients. Specifically, there was a trend toward longer overall operative times in men. This longer operative time was likely influenced by significantly longer mean times to dissect the hilum in men $(41 \mathrm{~min}) \mathrm{v}$ women (33 $\mathrm{min})(P=0.04)$ and also likely related to longer mean times to mobilize the kidney (men, 19 min; women, 15 $\min )(P=0.06)$.

A greater BMI was associated with a significantly longer mean operative time $\left(\mathrm{BMI}<25 \mathrm{~kg} / \mathrm{m}^{2}, 197 \mathrm{~min} ; \mathrm{BMI}=30\right.$, $225 \mathrm{~min})(P=0.03)$, which was predominantly related to the prolonged mean time to mobilize the colon $(\mathrm{BMI}<25$ $\mathrm{kg} / \mathrm{m}^{2}, 26 \mathrm{~min} ; \mathrm{BMI}=30,35 \mathrm{~min}(P<0.01)$.

We next evaluated which steps were most associated with the overall operative time of the procedure. Figure 1 depicts scatter plots demonstrating correlation (correlation coefficient, r) of individual surgical steps to overall operative time in patients undergoing HALDN. Colon mobilization and renal hilum dissection were most correlated with overall operative time. In addition, kidney mobilization and renal vein tributary dissection were also correlated with overall operative time, while kidney removal was not associated with overall operative time.

Table 1. Influence of Patient Factors on Operative Times

\begin{tabular}{|c|c|c|c|c|c|c|c|c|c|c|c|c|c|c|}
\hline Factor & Strata & $\mathrm{n}$ & Overall & $\mathrm{P}$ & Colon & $\mathrm{P}$ & Kidney & $\mathrm{P}$ & Vein & $\mathrm{P}$ & Hilum & $\mathrm{P}$ & Out & $\mathrm{P}$ \\
\hline \multirow{3}{*}{$\begin{array}{l}\text { Age } \\
\quad \text { (years) }\end{array}$} & $<30$ & 12 & 214 (39) & \multirow[t]{3}{*}{0.84} & $29(9)$ & \multirow[t]{3}{*}{0.38} & $16(7)$ & \multirow[t]{3}{*}{0.78} & $46(14)$ & \multirow[t]{3}{*}{0.82} & 37 (11) & \multirow[t]{3}{*}{0.07} & $15(9)$ & \multirow[t]{3}{*}{0.77} \\
\hline & $30-40$ & 17 & $211(36)$ & & $31(8)$ & & $18(10)$ & & 45 (14) & & 43 (19) & & $14(8)$ & \\
\hline & $>40$ & 39 & $207(34)$ & & 33 (1) & & $17(8)$ & & 43 (14) & & 33 (14) & & $13(7)$ & \\
\hline \multirow[t]{2}{*}{ Sex } & Man & 27 & 219 (37) & \multirow[t]{2}{*}{0.07} & $33(9)$ & \multirow[t]{2}{*}{0.23} & $19(8)$ & 0.06 & 44 (14) & \multirow[t]{2}{*}{0.95} & 41 (16) & \multirow[t]{2}{*}{0.04} & $14(8)$ & \multirow[t]{2}{*}{0.86} \\
\hline & Woman & 41 & $204(33)$ & & $31(9)$ & & $15(8)$ & & 44 (13) & & 33 (15) & & $14(7)$ & \\
\hline \multirow[t]{3}{*}{ BMI } & $<25$ & 14 & 197 (37) & \multirow[t]{3}{*}{0.03} & $26(8)$ & \multirow[t]{3}{*}{$<0.01$} & $15(10)$ & 0.64 & 47 (16) & \multirow[t]{3}{*}{0.24} & 34 (18) & \multirow[t]{3}{*}{0.92} & $13(6)$ & \multirow[t]{3}{*}{0.74} \\
\hline & $25-29$ & 29 & 205 (37) & & $32(10)$ & & $17(8)$ & & 42 (12) & & $35(14)$ & & $14(8)$ & \\
\hline & $\geq 30$ & 23 & $225(27)$ & & 35 (7) & & $18(9)$ & & 47 (12) & & $36(16)$ & & $14(8)$ & \\
\hline \multirow[t]{2}{*}{ ASA } & 1 & 45 & $212(32)$ & \multirow[t]{2}{*}{0.97} & $32(8)$ & \multirow[t]{2}{*}{0.55} & $17(9)$ & 0.79 & 44 (12) & \multirow[t]{2}{*}{0.58} & 37 (15) & \multirow[t]{2}{*}{0.97} & $15(8)$ & \multirow[t]{2}{*}{0.51} \\
\hline & 2 & 19 & $211(42)$ & & $33(11)$ & & $18(8)$ & & $46(16)$ & & 37 (15) & & $13(7)$ & \\
\hline \multirow{2}{*}{$\begin{array}{l}\text { Previous } \\
\text { surgery }\end{array}$} & Yes & 21 & 214 (27) & \multirow[t]{2}{*}{0.57} & $34(8)$ & \multirow[t]{2}{*}{0.15} & 19 (7) & 0.26 & 44 (11) & \multirow[t]{2}{*}{0.97} & 35 (13) & \multirow[t]{2}{*}{0.57} & $15(9)$ & \multirow[t]{2}{*}{0.76} \\
\hline & No & 44 & $208(39)$ & & $30(9)$ & & $16(9)$ & & 44 (15) & & 37 (17) & & $14(7)$ & \\
\hline \multirow{2}{*}{$\begin{array}{l}\text { Vascular } \\
\text { anatomy }\end{array}$} & Normal & 51 & 212 (37) & \multirow[t]{2}{*}{0.35} & $33(9)$ & 0.20 & $17(9)$ & 0.76 & 45 (13) & 0.63 & $36(15)$ & 0.91 & $14(8)$ & 0.92 \\
\hline & Abnormal & 17 & 203 (29) & & $29(9)$ & & $16(8)$ & & $43(15)$ & & $36(18)$ & & $14(6)$ & \\
\hline Side & Left & 64 & $210(36)$ & 0.73 & $32(9)$ & 0.43 & $17(8)$ & 0.93 & 45 (13) & 0.01 & $36(15)$ & 0.24 & $14(7)$ & 0.10 \\
\hline & Right & 4 & 204 (15) & & 35 (11) & & $17(8)$ & & $28(11)$ & & 45 (15) & & $8(4)$ & \\
\hline
\end{tabular}

$\mathrm{BMI}=$ body mass index in $\mathrm{kg} / \mathrm{m}^{2} ; \mathrm{ASA}=$ American Society of Anesthesilogists class; overall = overall operative times; colon = colon mobilization; kidney = kidney mobilization; vein = renal vein dissection and branches; hilum $=$ hilar dissection (including artery); out = removal of specimen. 



FIG. 1. Correlation of length of individual operative steps (minutes) to overall operative time (minutes). $r$ is the correlation coefficient. The $p$ value is the probability of the linear relationship occurring by chance.

The impact of increasing case experience on operative time is depicted in Fig. 2. There was flat but short operative times with increasing case experience for kidney removal. There was clear improvement with case experience for kidney/ ureteral mobilization in terms of shorter operative times with less variability. There was stagnant improvement for colon mobilization with continued wide variability in operative time despite increasing numbers of cases. This is in contrast to renal vein (and tributary) and hilum dissection which showed minimal changes in operative time over case experience but did show a decrease in variability. Overall operative time is reflective of the relatively stagnant improvement in operative times seen for individual surgical steps. However, like renal vein (and tributary) and hilum dissection, there appeared to be a reduction in variability over time.

To understand the impact of level of training on the relatively flat improvement in operative times, a multivariable model was created to compare the risk of a prolonged operative time by a junior $v$ senior resident for individual op- erative steps of HALDN (Table 2). Both the unadjusted and adjusted risks are described. For overall operative time, colon and kidney mobilization, there was a 3.5 to 6.4 times risk of a prolonged operative time when that step was performed by a junior resident $v$ a senior resident.

\section{Discussion}

Concerns over the diffusion of laparoscopic nephrectomy ${ }^{5,7}$ among urologists may be related to the methods by which the technology is disseminated. To that end, our study demonstrates several useful findings. While other studies have demonstrated the influence of patient factors on outcomes, ${ }^{8}$ our findings illuminate the patient factors that influence operative time and where these factors have their greatest impact. From our findings, it is possible that male gender and obesity may prolong operative time because of increases in intra-abdominal fat that make dissection more challenging. 
A



C



$\mathbf{E}$

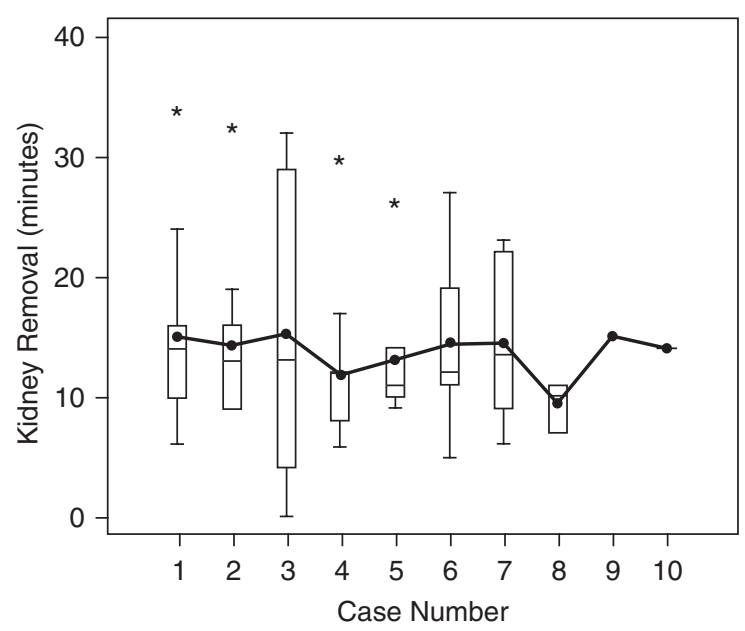

B
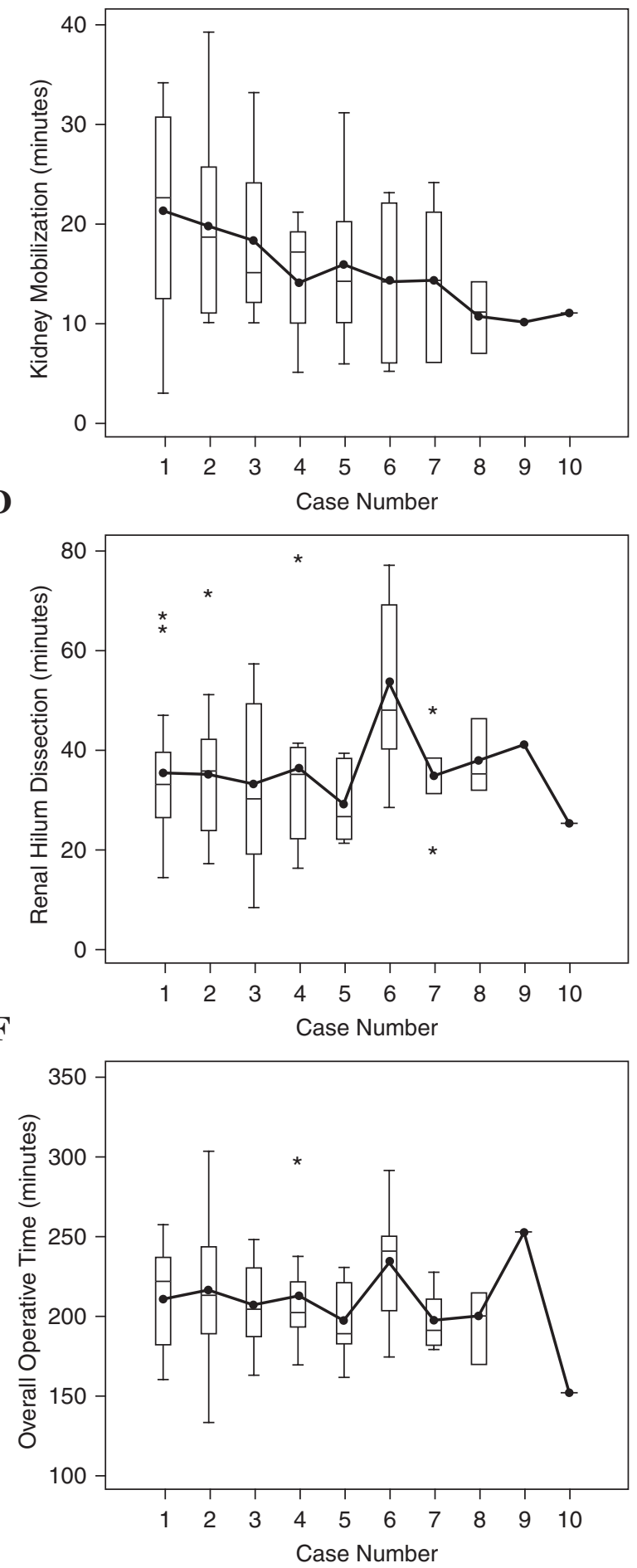

FIG. 2. Operative time by increasing case experience. Box plots represent the median (line in box), 25th percentile, 75th percentile with whiskers depicting maximum and minimum values. The bold line is drawn through mean values. Asterisks are outliers.

Our study also identified particular steps of the procedure that correlated most with overall operative time, which included colon mobilization and renal hilum dissection. When evaluating individual surgeons across individual steps, it was clear that both the level of training as well as the complexity of the step influenced the rate at which surgeons' operative times improved for that portion of the procedure.
While these findings are interesting, there are some limitations to our current study. This was a small study of only 70 patients. While the patient number was small, each patient's procedure, however, was broken into six different time points. which provided 420 evaluable measures associated with an individual surgeon and created a robust dataset. 
Another potential limitation is the fact that our study attempts to draw conclusions about laparoscopic nephrectomy in general from laparoscopic donor nephrectomy cases. While not the same procedure, most of the steps are similar between the HALDN and hand-assisted laparoscopic nephrectomy, with variation in the order for donor nephrectomy to limit warm ischemia time. Therefore, this model is useful to assess learning of laparoscopic nephrectomy.

Because the attending surgeon limited which steps could be performed by a surgeon based on level of training, there are some difficulties in using this dataset to draw conclusions regarding the impact of level of training on learning HALDN. As can be seen from Table 2, however, there is enough overlap between different levels of training and surgical steps to demonstrate the impact of the surgeon level factor on operative times.

A final limitation of our study is in the use of operative time as an outcome measure. While improvement in operative time suggests a surgeon is learning the procedure, it is only a single piece of what constitutes a surgeon's competence. We did not evaluate other factors, such as surgical complications, graft function, patient recovery, or other more difficult factors to measure (surgeon comfort with anatomy, ability to recognize problems and change surgical plan, etc), because each procedure was performed with the assistance of multiple surgeons and therefore these measures could not be assessed individually. These factors, however, may provide a more global assessment of a surgeon as we seek to identify barriers to urologists performing laparoscopic nephrectomy. The use of surgical simulators is an attempt to evaluate a trainees overall competence. ${ }^{9}$

How do these findings help address the initial problem? We believe that there are several findings in this study that can provide guidance in improving diffusion of laparoscopic nephrectomy. While a practicing surgeon cannot select the patient characteristics of the patients coming to his or her office with renal masses, a teaching surgeon can be cognizant of unfavorable patient characteristics when guiding residents and trainees through individual procedures.

The two more controllable points for those training persons to perform laparoscopic nephrectomy are that certain parts of the procedures correlate with mastery of the procedure (and therefore need extra attention in teaching) and previous experience (even if not laparoscopic) does help a surgeon in learning the procedure. For residents, dedicated exposure to laparoscopy at multiple points during residency may provide the necessary experience to master these skills. ${ }^{10,11}$ For those residents without exposure to laparoscopy during training, some amount of fellowship training may be important to master these skills.

For practicing urologists, postgraduate training needs to be more focused on the key portions of the procedure to create comfort in those surgeons to take their training back and provide care to patients. In addition, as suggested by others, this training (typically in animal laboratories and didactic sessions) is best followed up by proctoring by an experienced surgeon. This follow-up has been shown to result in a trainee who is more likely to perform laparoscopy long term. ${ }^{12}$

Alternatively, a mini-fellowship in laparoscopic skills may provide the didactic training (lectures) and technical experience (animal laboratories, surgical assistance, and proctored surgery) to enable practicing urologists to implement these tools into their practice without a high opportunity cost (fellowship training). It would also afford the specialty a way of controlling and assessing the quality of practitioners who are interested in offering advanced surgical techniques to patients. These short fellowships have been shown to result in the successful and durable transfer of laparoscopic skills to the practicing urologist. ${ }^{13,14}$

A final alternative is the use of surgical simulators to train surgeons in certain technical skills. While these have been shown to have some benefit in laparoscopic procedures, it is likely less effective than clinical training and cannot be the complete solution to training practicing urologists. ${ }^{15}$ While these simulators will increasingly be able to replicate the surgical environment in the future, they are unlikely to replace the "hands-on" training needed for the current practitioner. They appear to be more useful in assessing competence and providing the initial introduction to new skills.

\section{Conclusions}

While laparoscopy has been shown to have improved benefits over open surgery, the diffusion of this skill has lagged behind its acquisition in other specialties. Our study demonstrates that patient characteristics, particular surgical steps, and the training level of the surgeon may all impact the learning of HALDN. Resident and postgraduate training needs to address these shortfalls with more dedicated exposure to this approach either during training or through the use of "mini-fellowships" for postgraduate trainees. This approach will move beyond the "see one, do one, teach one" philosophy and potentially result in greater diffusion of this skill and improved patient safety.

Table 2. Risk of Longer Operative Time by Junior versus Senior Resident Adjusted for Patient Factors

\begin{tabular}{lccccc}
\hline & \multicolumn{2}{c}{ Unadjusted risk } & & \multicolumn{2}{c}{ Adjusted risk } \\
\cline { 2 - 3 } \cline { 5 - 6 } Operative time & $\begin{array}{c}\text { Odds } \\
\text { ratio }\end{array}$ & $\begin{array}{c}95 \% \text { confidence } \\
\text { interval }\end{array}$ & & $\begin{array}{c}\text { Odds } \\
\text { ratio }\end{array}$ & $\begin{array}{c}\text { 95\% confidence } \\
\text { interval }\end{array}$ \\
\hline Overall operative time & 2.5 & $0.9-6.6$ & 5.9 & $1.3-27.7$ \\
Colon mobilization & 2.5 & $0.9-6.6$ & 3.5 & $0.9-14.5$ \\
Kidney/ureter mobilization & 2.5 & $0.9-6.9$ & 6.4 & $1.5-27.0$ \\
\hline
\end{tabular}

Adjusted for body mass index and patient sex. 


\section{References}

1. Jarrett TW, Chan DY, Charambura TC, Fugita O, Kavoussi LR. Laparoscopic pyeloplasty: The first 100 cases. J Urol 2002;167:1253-1256.

2. Portis AJ, Yan Y, Landman J, et al. Long-term followup after laparoscopic radical nephrectomy. I Urol 2002;167: 1257-1262.

3. Wolf JS Jr, Merion RM, Leichtman AB, Campbell DA Jr, Magee JC, Punch JD, Turcotte JG, Konnak JW. Randomized controlled trial of hand-assisted laparoscopic versus open surgical live donor nephrectomy. Transplantation 2001; 72:284-290.

4. Permpongkosol S, Chan DY, Link RE, et al. Long-term survival analysis after laparoscopic radical nephrectomy. $\underline{\text { J Urol }}$ 2005;174:1222-1225.

5. Miller DC, Wei JT, Dunn RL, Hollenbeck BK. Trends in the diffusion of laparoscopic nephrectomy. IAMA 2006;295: 2480-2482.

6. Morris DS, Miller DC, Hollingsworth JM, Dunn RL, Roberts WW, Wolf JS Jr, Hollenbeck BK. Differential adoption of laparoscopy by treatment indication. J Urol 2007;178:2109-2113.

7. Miller DC, Taub DA, Dunn RL, Wei JT, Hollenbeck BK. Laparoscopy for renal cell carcinoma: diffusion versus regionalization? J Urol 2006;176:1102-1107.

8. Anast JW, Stoller ML, Meng MV, Master VA, Mitchell JA, Bassett WW, Kane CJ. Differences in complications and outcomes for obese patients undergoing laparoscopic radical, partial or simple nephrectomy. I Urol 2004;172: 2287-2291.

9. McDougall EM, Corica FA, Boker JR, Sala LG, Stoliar G, Borin JF, Chu FT, Clayman RV. Construct validity testing of a laparoscopic surgical simulator. I Am Coll Surg 2006; 202:779-787.

10. Gaston KE, Moore DT, Pruthi RS. Hand-assisted laparoscopic nephrectomy: Prospective evaluation of the learning

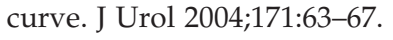

11. Hollenbeck BK, Seifman BD, Wolf JS Jr. Clinical skills acquisition for hand-assisted laparoscopic donor nephrectomy. J Urol 2004;171:35-39.

12. Marguet CG , Young MD, L'Esperance JO, Tan YH, Ekeruo WO, Preminger GM, Albala DM. Hand assisted laparoscopic training for postgraduate urologists: The role of mentoring. J Urol 2004;172:286-289.

13. Corica FA, Boker JR, Chou DS, et al. Short-term impact of a laparoscopic "mini-residency" experience on postgraduate urologists' practice patterns. J Am Coll Surg 2006;203:692-698.

14. Shalhav AL, Dabagia MD, Wagner TT, Koch MO, Lingeman JE. Training postgraduate urologists in laparoscopic surgery: The current challenge. I Urol 2002;167:2135-2137.

15. Andreatta PB, Woodrum DT, Birkmeyer JD, Yellamanchilli RK, Doherty GM, Gauger PG, Minter RM. Laparoscopic skills are improved with LapMentor training: Results of a randomized, double-blinded study. Ann Surg 2006;243:854-863.

Address reprint requests to: Alon Z. Weizer, MD Department of Urology University of Michigan TC 3875 Box 5330

1500 E. Medical Center Drive Ann Arbor, MI 48109

E-mail: aweizer@umich.edu
Abbreviations Used
ASA $=$ American Society of Anesthesiologists
$\mathrm{BMI}=$ body mass index
HALDN = hand-assisted laparoscopic donor nephrectomy. 